\title{
Urinary tartaric acid as a potential biomarker for the dietary assessment of moderate wine consumption: a randomised controlled trial
}

\author{
Jorge Regueiro $^{1}$, Anna Vallverdú-Queralt ${ }^{2,3}$, Jesús Simal-Gándara ${ }^{1}$, Ramón Estruch ${ }^{3,4}$ and \\ Rosa María Lamuela-Raventós ${ }^{2,3 *}$ \\ ${ }^{1}$ Nutrition and Bromatology Group, Analytical and Food Chemistry Department, Faculty of Food Science and \\ Technology, Ourense Campus, University of Vigo, Ourense 32004, Spain \\ ${ }^{2}$ Department of Nutrition and Food Science, XaRTA, INSA, School of Pharmacy, University of Barcelona, \\ Avinguda Joan XXIII s/n, Barcelona 08028, Spain \\ ${ }^{3}$ Centro de Investigación Biomédica en Red de Fisiopatología de la Obesidad y Nutrición (CIBERObn), \\ Instituto de Salud Carlos III, Madrid, Spain \\ ${ }^{4}$ Department of Internal Medicine, Hospital Clinic, Institut d'Investigació Biomèdica August Pi i Sunyer (IDIBAPS), \\ University of Barcelona, Barcelona, Spain
}

(Submitted 4 July 2013 - Final revision received 11 November 2013 - Accepted 15 November 2013 - First published online 10 February 2014)

\begin{abstract}
The availability of biomarkers that allow the estimation of the intake of specific foods and dietary components, as an alternative or addition to self-reported dietary questionnaires, could greatly enhance the effectiveness of nutritional research. The aim of the present study was to assess tartaric acid, one of the major components of red and white wines, as a potential biomarker of wine consumption. A total of twentyone healthy men participated in a randomised cross-over feeding trial. They consumed a single dose of 100,200 or $300 \mathrm{ml}$ wine at dinner. Before each intervention, the participants followed a $7 \mathrm{~d}$ washout period during which they avoided consuming wine or grape-based products. Morning urine was collected and analysed by liquid chromatography coupled to electrospray ionisation tandem MS. A strong significant correlation was found between wine intake and urinary tartaric acid $\left(r_{\mathrm{s}}=0.9220 ; P<0 \cdot 001\right)$. Using a cut-off value of $8.84 \mu \mathrm{g} / \mathrm{mg}$ creatinine, tartaric acid allowed wine consumers to be differentiated from non-wine consumers. The results suggest that urinary tartaric acid may be a sensitive and specific dietary biomarker of wine consumption.
\end{abstract}

Key words: Biomarker: Grapes: Tartaric acid: Urine: Wine

Some of the disadvantages associated with self-reported dietary questionnaires in epidemiological and clinical studies ${ }^{(1,2)}$ can be overcome by using biomarkers that provide a more objective assessment of nutrient intake ${ }^{(3-5)}$. In the case of wine, a biomarker of intake might reflect consumption more accurately than a questionnaire, as individuals may be reluctant to accurately report customary drinking level due to the social undesirability of excessive alcoholic beverage consumption ${ }^{(6)}$.

Total urinary resveratrol metabolites (TRM) have been proposed as biomarkers of wine intake in both clinical and epidemiological studies ${ }^{(7,8)}$, although the high variation in resveratrol content between red and white wines, as a result of different oenological practices, and also between grape varieties $^{(9,10)}$, is an important limitation. In addition, the varying microbiological metabolism reported for resveratrol ${ }^{(11)}$ could contribute to a higher variation. Therefore, to obtain greater insight into the health effects of moderate wine drinking, new reliable biological markers for wine intake are needed.

In this context, tartaric acid might be a promising candidate, as it accounts for a major fraction of wines, normally within the range of $1.5-4.0 \mathrm{~g} / \mathrm{l}^{(12,13)}$. Tartaric acid, the main acid responsible for wine acidity, is a natural organic acid occurring in grapes at a high concentration (up to $1 \%$ of whole fresh grapes), but rare in most other common plants ${ }^{(13,14)}$. This fact provides high selectivity for tartaric acid when compared with other wine constituents. Furthermore, tartaric acid is not degraded during the winemaking process, and hence its levels remain relatively constant in both red and white wines ${ }^{(13)}$. Studies on its metabolism in human subjects have suggested that tartrate excreted in the urine is mainly from dietary sources.

Abbreviations: LC-ESI-MS/MS, liquid chromatography coupled to electrospray ionisation tandem MS; ROC, receiver-operating characteristic; TRM, total resveratrol metabolites.

*Corresponding author: R. M. Lamuela-Raventós, fax +3493 4035931, email lamuela@ub.edu 
Unlike other fruit acids (such as malic and citric acids), which are digested and converted to energy in the body, tartaric acid mostly bypasses the small intestine and is fermented by colonic bacteria, and has been shown to have a beneficial role in intestinal function ${ }^{(15)}$.

Lord et $a l{ }^{(16)}$ reported a significant increase in urinary tartaric acid after consumption of grape juice, while VázquezFresno et al. ${ }^{(17)}$ observed that its urinary concentrations were significantly higher in wine consumers than in non-wine consumers.

Nevertheless, the use of urinary tartaric acid as a biomarker of wine consumption has not been formally assessed. Therefore, we evaluated the usefulness of tartaric acid as a wine biomarker in a randomised, controlled, cross-over clinical trial conducted in healthy male volunteers.

\section{Materials and methods}

\section{Reagents and standards}

L-(+)-Tartaric acid and creatinine were purchased from Sigma. The labelled internal standard DL- $( \pm)$-tartaric-2,3-d2 acid was obtained from $\mathrm{C} / \mathrm{D} / \mathrm{N}$ Isotopes. Formic acid (approximately 98\%), picric acid (98\%, moistened with approximately 33\% water) and sodium hydroxide ( $\geq 98 \%)$ were purchased from Panreac. Solvents were HPLC grade and all other chemicals were analytical reagent grade. Ultrapure water was obtained from a Milli-Q Gradient water purification system (Millipore).

Stock solutions of tartaric acid were prepared in water. Different working standard solutions were made by appropriate dilution in $0.5 \%$ formic acid in water and then stored in amber glass vials at $-20^{\circ} \mathrm{C}$.

\section{Wine samples}

A commercial red wine from the Spanish Protected Designation of Origin 'Rioja' was used over the course of the present study. It was elaborated with grapes of Vitis vinifera $\mathrm{L}$. and consisted of a blend of Tempranillo (85\%) and Graciano and Garnacha Tinta (15\%) varieties from the 2009 vintage. To ensure its homogeneity, the wine belonged to the same batch and was bottled on the same day. The wine had a pH value of 3.7 and $13.0 \%$ alcohol by volume. The content of tartaric acid was determined by liquid chromatography coupled to electrospray ionisation tandem MS (LC-ESI-MS/MS) as described previously ${ }^{(18)}$. Tartaric acid level in this wine was 1737 (sD 17) mg/l ( $n$ 3).

\section{Subjects and study design}

A total of twenty-one Caucasian healthy men, with a mean age of 30.7 (SD 5.9) years (median 30.0 years; range $21-50$ years) and a mean BMI of $24.7(\mathrm{SD} 2.7) \mathrm{kg} / \mathrm{m}^{2}$ (median $24.2 \mathrm{~kg} / \mathrm{m}^{2}$; range $19 \cdot 8-32.9 \mathrm{~kg} / \mathrm{m}^{2}$ ), participated in a randomised crossover feeding trial

The subjects were non-smokers; had no history of cardiovascular, hepatic or renal disease; had no acute or chronic infection, inflammatory disease or endocrine disorders; had no anti-inflammatory, corticosteroid, hormonal or antibiotic drug treatment during the previous 3 months; had no history of alcohol abuse or drug dependence; and had not adhered to any special diets at least 4 weeks before the commencement of the study. Only male subjects were recruited, in order to reduce the menstrual cycle phase-related variability in women, putatively affecting the absorption, metabolism and excretion processes.

The present study was conducted according to the guidelines laid down in the Declaration of Helsinki, and all procedures involving human subjects were approved by the Ethics Committee of Clinical Investigation of the University of Barcelona (Spain) (reference no. IRB0003099). Written informed consent was obtained from all subjects. The study protocol followed the recommendations made in the CONSORT (Consolidated Standards of Reporting Trials) guidelines ${ }^{(19)}$. The present trial was registered at controlledtrials.com as ISRCTN63399546.

The interventions consisted of intake at dinner, in a random order, of 100,200 and $300 \mathrm{ml}$ red wine, corresponding to $10 \cdot 3,20.5$ and $30.8 \mathrm{~g}$ ethanol, respectively. The participants were randomly assigned following simple randomisation procedures (computerised random numbers) to one of three treatment groups. Before each intervention, the participants followed a $7 \mathrm{~d}$ washout period during which they were requested to avoid consuming wine or grape-based products.

First morning urine samples were collected the day before the first intervention and in the morning following each intervention (Fig. 1). All samples were collected in $100 \mathrm{ml}$ random coded sterile specimen containers, and immediately stored at $4^{\circ} \mathrm{C}$. Upon receipt of each sample, four aliquots of $1.0 \mathrm{ml}$ were transferred to separate $1.5 \mathrm{ml}$ capped Eppendorf tubes and stored at $-80^{\circ} \mathrm{C}$ until the analyses, which were performed with no knowledge of the clinical data.

\section{Analytical methods}

Determination of urinary tartaric acid concentration was carried out according to a previously validated stable-isotope dilution LC-ESI-MS/MS method ${ }^{(18)}$. Briefly, urine samples $(50 \mu \mathrm{l})$ were diluted $1: 20(\mathrm{v} / \mathrm{v})$ with $0.5 \%$ formic acid in water, and $10 \mu \mathrm{l}$ of a ${ }^{2} \mathrm{H}$-labelled isotope standard solution in water (DL- $( \pm)$ tartaric-2,3-d2 acid, $60 \mu \mathrm{g} / \mathrm{ml}$ ) were added. The sample dilution was filtered using a $0.20 \mu \mathrm{m}$ filter and analysed by LC-ESI-MS/MS. All samples were analysed in triplicate.

To normalise analyte concentrations in spot urine samples, all results were corrected for urinary creatinine ${ }^{(20)}$, and are reported as $\mu \mathrm{g}$ tartaric acid/mg creatinine in the morning urine. Urinary creatinine was determined by a modification of Jaffé's alkaline picrate method ${ }^{(21)}$.

LC-ESI-MS/MS analyses were performed on an Agilent 1100 Series HPLC system (Agilent Technologies) coupled to a triple quadrupole mass spectrometer API 3000 (Applied Biosystems) as described elsewhere ${ }^{(18)}$. Chromatographic separation was performed on a reversed-phase column Atlantis T3 C18 $(100 \times 2.1 \mathrm{~mm}, 3 \mu \mathrm{m})$ from Waters maintained at $25^{\circ} \mathrm{C}$. Mobile phases A and B were, respectively, 0.5\% formic acid in water and $0.5 \%$ formic acid in acetonitrile. The following linear gradient was used: held at $100 \%$ A for $3.5 \mathrm{~min}$, 


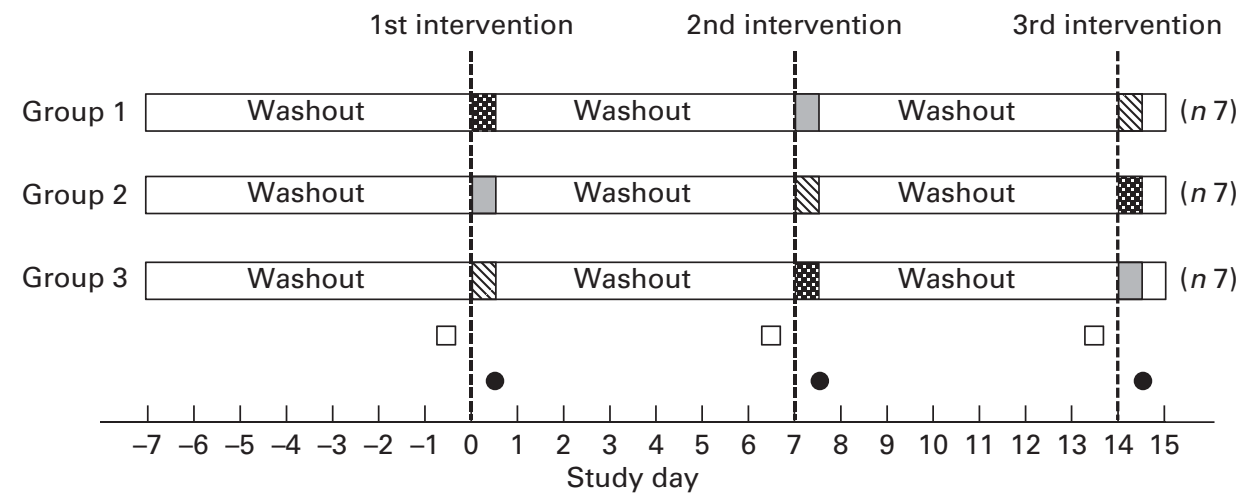

Fig. 1. Schematic representation of the study design. $\mathbb{\mathbb { N }}$, Consumption of $100 \mathrm{ml}$ wine at dinner; $\square$, consumption of $200 \mathrm{ml}$ wine at dinner; $\mathbf{0}$, consumption of $300 \mathrm{ml}$ wine at dinner; $\square$, basal urine sample; $\bullet$, post-intervention urine sample.

decreased to $10 \% \mathrm{~A}$ over $2 \mathrm{~min}$ and held for $2 \mathrm{~min}$, and then returned to initial conditions for $1.5 \mathrm{~min}$ and re-equilibrated for $6 \mathrm{~min}$. The flow rate was set at $350 \mu \mathrm{l} / \mathrm{min}$ and the injection volume was $10 \mu \mathrm{l}$. Post-column addition of acetonitrile ( $250 \mu \mathrm{l} / \mathrm{min}$ ) was carried out to improve analyte ionisation efficiency. Detection was accomplished in the multiple reaction monitoring mode, and the following MS/MS transitions were used for quantification and confirmation, respectively: $m / z 149 / 87$ and $m / z 149 / 73$ for tartaric acid, and $m / z 151 / 88$ and $m / z 151 / 74$ for the ${ }^{2} \mathrm{H}$-labelled isotope.

\section{Statistical analysis}

Statistical calculations were made using the software package GraphPad Prism version 5.0 (GraphPad Software). Unless otherwise specified, data are presented as means and standard deviations, and a 0.05 significance level was used. All data were assessed for normality using the 'omnibus K2' D'AgostinoPearson and the Shapiro-Wilk normality tests. Since concentration of urinary tartaric acid was not normally distributed, the non-parametric Mann-Whitney $U$ test and Wilcoxon test were applied to determine statistical difference between groups. Spearman's rank correlation was used to estimate the relationship between urinary excretion and wine consumption. Receiver-operating characteristic (ROC) curve analysis was performed on the concentration of urinary tartaric acid to evaluate its sensitivity and specificity in the discrimination between periods of abstention and wine drinking. To determine the optimal cut-off value, ROC curves were constructed using all possible cut-off points for each assay. For sensitivity and specificity, 95\% CI were calculated using the Clopper-Pearson exact binomial method.

\section{Results}

The estimated intake of tartaric acid for the three interventions was 174 (SD 2), 347 (SD 3) and 521 (SD 5) mg for 100, 200 and $300 \mathrm{ml}$ wine consumption, respectively.

The concentrations of urinary tartaric acid before and after each intervention are shown in Fig. 2. After the $7 \mathrm{~d}$ washout periods, the median urinary concentration was 1.06 (interquartile range $0.56-1.78) \mu \mathrm{g} / \mathrm{mg}$ creatinine. After wine consumption, a significant rise in its urinary concentration was observed. The amount of tartaric acid increased by 27.0 (95\% CI 22.9 , $32.9) \mu \mathrm{g} / \mathrm{mg}(P<0.001)$ after the intake of $100 \mathrm{ml}$ wine, by 52.5 $(95 \%$ CI $44.5,69.7) \mu \mathrm{g} / \mathrm{mg}(P<0.001)$ after the intake of $200 \mathrm{ml}$ wine and by $80.8(95 \%$ CI $73.1,107) \mu \mathrm{g} / \mathrm{mg}(P<0.001)$ after the intake of $300 \mathrm{ml}$ wine. The order of interventions did not affect the results. Spearman's correlation analysis revealed a strong direct association between wine dose and urinary tartaric acid $\left(r_{\mathrm{s}}=0.9220 ; P<0.001\right)$.

To assess the effectiveness of tartaric acid as a biomarker of wine intake, a ROC curve was constructed, showing an AUC of $100 \%(P<0.001$; Fig. 3). The optimal cut-off point was $8.84 \mu \mathrm{g} / \mathrm{mg}$ creatinine $(58.9 \mathrm{nmol} / \mathrm{mg}$ creatinine $)$ which allowed wine consumers to be differentiated from non-wine consumers. This point had a sensitivity of $100 \%(95 \% \mathrm{CI}$ $83.9,100 \%)$ and a specificity of $100 \%$ (95\% CI 83.9, 100\%). When trying to differentiate between the low dose $(100 \mathrm{ml})$ and the intermediate dose $(200 \mathrm{ml})$, the resulting ROC curve presented an AUC of $91.4 \%$ (95\% CI 82.6, $100 \%$; $P<0.001$ ) and an optimal cut-off value of $40.7 \mu \mathrm{g} / \mathrm{mg}$ creatinine

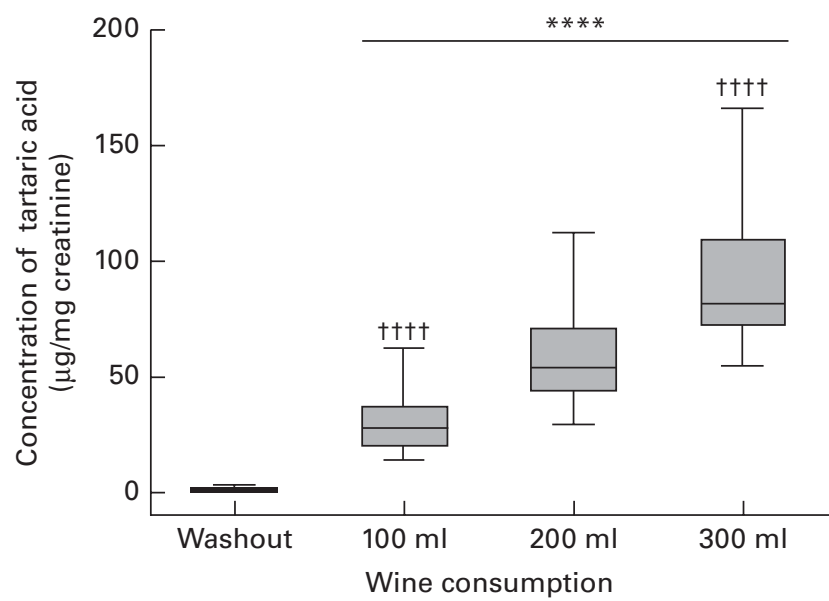

Fig. 2. Box and whisker plot showing the median (central line), 25-75 percentiles (boxes) and the entire range (whiskers) for the urinary tartaric acid concentration after the $7 \mathrm{~d}$ washout periods and after the intake of 100 , 200 and $300 \mathrm{ml}$ wine, respectively. ${ }^{* \star \star \star}$ Mean value was significantly different from that of the washout period $(P<0.0001)$. ††††Mean value was significantly different from that of the $200 \mathrm{ml}$ intake $(P<0.0001)$. 


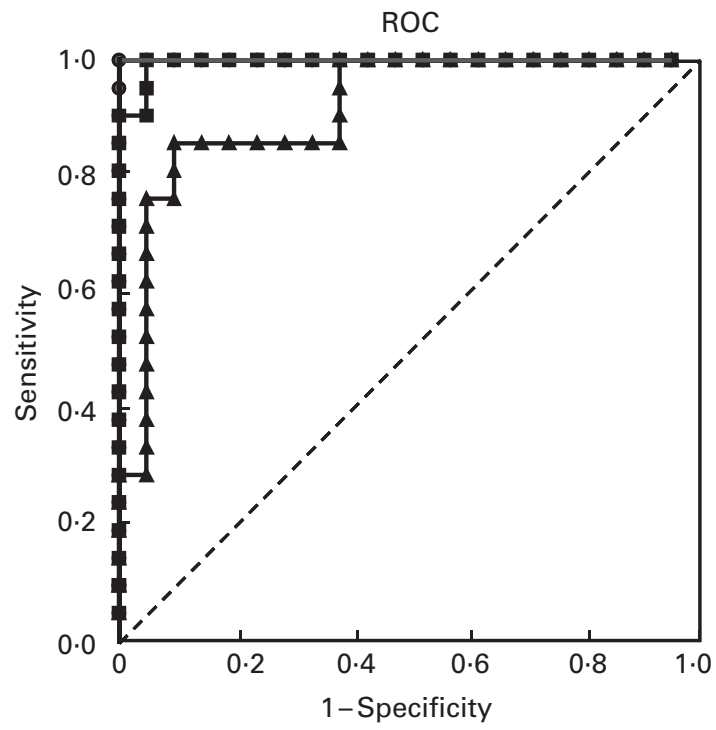

Fig. 3. Receiver-operating characteristic $(\mathrm{ROC})$ curves of urinary tartaric acid to discriminate between wine and non-wine consumers, and between low $(100 \mathrm{ml})$ and moderate $(300 \mathrm{ml})$ wine consumption in the clinical trial. -o-, Non-consumers v. $100 \mathrm{ml}$ wine intake; $\_$, 100 v. $200 \mathrm{ml}$ wine intake; $\rightarrow, 100 \mathrm{v.} 300 \mathrm{ml}$ wine intake.

( $271 \mathrm{nmol} / \mathrm{mg}$ creatinine), which resulted in a sensitivity of $85.7 \%(95 \%$ CI $63.7,96.9 \%)$ and a specificity of $90.5 \%$ (95\% CI 69.6, 98.8\%). Another ROC curve was obtained, aiming to differentiate between the intake of 100 and $300 \mathrm{ml}$ wine (Fig. 3). In this case, the AUC was 99.6\% (95\% CI 98.3, $100 \% ; P<0.001)$ and the optimal cut-off value was $48.7 \mu \mathrm{g} / \mathrm{mg}$ creatinine $(324 \mathrm{nmol} / \mathrm{mg}$ creatinine). This point had a sensitivity of $100 \%(95 \%$ CI $83.9,100 \%)$ and a specificity of $95 \cdot 2 \%(95 \%$ CI $76 \cdot 2,99 \cdot 9 \%)$

\section{Discussion}

A major challenge for scientists investigating the healthpromoting effects of moderate wine consumption is to reliably and accurately assess the consumption status in their clinical and/or epidemiological studies.

In this regard, concentration of urinary TRM has been previously proposed as a biomarker of wine intake. TRM showed a significant dose-response effect ( $r$ 0.645; $P<0.001)$, and it was able to differentiate between wine drinkers and non-wine drinkers with high sensitivity and specificity (72 and 94\%, respectively) at a cut-off value of $90 \mathrm{nmol} / \mathrm{g}$ creatinine ${ }^{(8)}$. Nevertheless, TRM has limitations as a biomarker because resveratrol is not specific to grapes and can also be found in peanuts, although at lower concentrations $^{(22,23)}$; moreover, resveratrol content has been shown to vary substantially among different types of wine ${ }^{(9,22)}$

Further research is therefore needed to evaluate other wine constituents or their metabolites in the human body as potential biomarkers of wine consumption. The aim of the present study was to examine whether tartaric acid, a main wine component, might be a suitable biomarker of moderate wine consumption. In contrast to resveratrol, with several important dietary sources, the major source of tartaric acid in the diet is grapes, and therefore also wine ${ }^{(13,14)}$. Although it can be found in other fruits such as bananas, its concentration levels can be considered negligible when compared with grapes or wine ${ }^{(14,24)}$. Only tamarind, a sour fruit whose consumption is mainly restricted to tropical areas, presents comparable contents of this $\operatorname{acid}^{(15)}$.

Accordingly, after the washout periods, during which the only dietary restriction was the consumption of wine or any other grape-based products, a very low concentration of tartaric acid was detected in the urine, probably due to the previous intake of some food containing low levels of tartaric acid.

An effective nutritional biomarker should also be robust and have a strong direct relationship with specific dietary consumption $^{(25,26)}$. The strong correlation observed between wine intake and urinary tartaric acid indicates that the concentration of this acid in the urine reflects the amount of wine consumed. Thus, its urinary concentration underwent a 27-fold increase after drinking only $100 \mathrm{ml}$ wine and almost 80 -fold after consumption of $300 \mathrm{ml}$ wine. In a previous study conducted in five male volunteers, the concentration of urinary tartaric acid also significantly increased $(P<0.01)$ after consumption of $200 \mathrm{ml}$ red wine, reaching urinary levels between 35.1 and $91.8 \mu \mathrm{g} / \mathrm{mg}$ creatinine ${ }^{(18)}$. Similar results have been reported by Lord et al. ${ }^{(27)}$ in urine samples of two healthy men after the consumption of two glasses of wine, which showed an increase in tartaric acid concentrations from $<10 \mu \mathrm{g} / \mathrm{mg}$ to 179 and $210 \mu \mathrm{g} / \mathrm{mg}$, respectively. In a further study with twenty-three subjects, the same authors observed the dietary impact of grape juice consumption on urinary tartrate, which rose from 7.40 to $282 \mu \mathrm{g} / \mathrm{mg}$ after the intake of $280 \mathrm{ml}$ grape juice $^{(16)}$. More recently, Vázquez-Fresno et $a l^{(17)}$ also reported an increase in urinary tartaric acid concentrations in sixty-one male volunteers with high cardiovascular risk factors after the intake of $272 \mathrm{ml}$ red wine and dealcoholised red wine.

Since this acid comes directly from grapes, their consumption as table grapes, raisins or processed into jams and juices would also increase its urinary concentration in a similar way to wine. However, most grapes are used for winemaking (approximately $70 \%$ ), about 30\% are consumed fresh and only a minor portion $(<1 \%)$ are consumed as raisins ${ }^{(28)}$.

To the best of our knowledge, the influence of the food matrix on the bioavailability of tartaric acid has not yet been studied. Interestingly, with the exception of wine or grapebased products, no restrictions were made regarding the intake of food and beverages on the previous days or even on the day of the intervention. Thus, the present results were obtained under real feeding conditions, which suggested that the absorption and excretion of tartaric acid is little affected by the food matrix. This finding confirms that this potential biomarker of wine intake would be selective enough to be minimally affected by a general diet.

Another requirement is that the biomarker permits the discrimination between different levels of consumption ${ }^{(29)}$. Under controlled trial conditions, a cut-off value of $8.84 \mu \mathrm{g} / \mathrm{mg}$ creatinine $(58.9 \mathrm{nmol} / \mathrm{mg}$ creatinine) in the concentration of urinary tartaric acid allowed consumers and non-consumers of wine to be distinguished with maximal sensitivity and 
specificity (Fig. 3). A second ROC curve was constructed aiming to differentiate between the wine intakes of the low dose $(100 \mathrm{ml})$ and the intermediate dose $(200 \mathrm{ml})$, obtaining a cut-off value of $40.7 \mu \mathrm{g} / \mathrm{mg}$ creatinine $(271 \mathrm{nmol} / \mathrm{mg}$ creatinine), showing enough sensitivity and specificity. Finally, a cut-off value of $48.7 \mu \mathrm{g} / \mathrm{mg}$ creatinine ( $324 \mathrm{nmol} / \mathrm{mg}$ creatinine) was established to distinguish between low-dose $(100 \mathrm{ml})$ and moderate-dose wine consumers $(300 \mathrm{ml})$, again showing maximal sensitivity and specificity $>95 \%$.

It should be noted that the present data were obtained from a randomised, controlled, cross-over study in which individuals acted as their own controls, which are the optimal conditions for investigating dietary biomarkers. Furthermore, these results might not be generalisable to the women population as only men were included in the present study. Therefore, further research with free-living populations is necessary to determine whether the excretion of tartaric acid can categorise individuals, both men and women, according to their wine intake under uncontrolled conditions.

In comparison with urinary resveratrol metabolites, urinary tartaric acid has some potential advantages. In this context, one of the major limitations of using resveratrol metabolites is the highly varying concentration of resveratrol in wine, usually within the range of $0.98-18.0 \mathrm{mg} / \mathrm{l}$ depending on the grape variety ${ }^{(22)}$. In addition, the concentration of resveratrol in red wines is much higher than that in white wines, since during the red winemaking, skins, which account for the highest content of resveratrol in grapes ${ }^{(9,30)}$, are macerated with the juice, while in white winemaking, only the free-run juice without skin contact is used.

Furthermore, it has been shown that colonic microbiota may play a significant role in resveratrol metabolite production, which needs further investigation due to high inter-individual human microbiota variability ${ }^{(11)}$.

In contrast, the concentration of tartaric acid in different wines is far more constant, normally within the range of $1 \cdot 5-4.0 \mathrm{~g} / \mathrm{l}^{(12,13)}$. Tartaric acid is mainly present in the grape pulp, which is used for the winemaking of all kinds of wine, so it would seem more suitable than TRM as a biomarker of wine consumption, including wines with lower contents of resveratrol, such as rose and white wines. Another important advantage relies on the much higher concentration of tartaric acid in wine compared with resveratrol, usually more than 1000-fold. This fact, along with the relatively high percentage of tartrate excreted unchanged in the urine, $14-20 \%$ of ingested dose in human subjects $^{(31)}$, would be responsible for the high concentrations of tartaric acid in the urine after wine consumption. This enables a faster sample preparation by simple urine dilution. From an analytical point of view, it results in a reduction of sample manipulation and cost, and the same time increasing sustainability, accuracy and precision in biomarker measurement.

On the basis of the present findings, urinary tartaric acid may be considered as a sensitive, selective and robust biomarker of moderate wine intake. The present study design did not consider the potential of this acid as a long-term biomarker of intake, especially if wine is episodically consumed. It is likely that tartaric acid is a good short-term biomarker, and that a single urine sample may not be sufficient to assess 'usual' intake.
Nevertheless, this biomarker could be used in studies in which repeated samples are available and also in combination with self-reported questionnaires to improve the accuracy of intake assessment. The next step is to evaluate whether it can improve the accuracy of intake assessment in epidemiological studies, where the conditions of the participants are less well controlled ${ }^{(32)}$.

\section{Acknowledgements}

The present study was financially supported by Junta de Andalucía and the Spanish Ministry of Economy and Competitiveness funds (AGL2010-22319-C03-01/02) and CIBEROBN. J. R. and A.V.-Q. were supported by contracts Juan de la Cierva and FPU, respectively, from the Spanish Ministry of Science and Innovation. Junta de Andalucía and the Spanish Ministry of Economy and Competitiveness had no role in the design, analysis or writing of this article.

The authors' responsibilities were as follows: J. S.-G., R. E. and R. L.-R. designed the research; J. R. and A. V.-Q. designed the analytical approach; R. E. and R. L.-R. designed the intervention study; J. R., A. V.-Q., R. E. and R. L.-R. recruited the participants and collected the metadata; J. R. and A. V.-Q. performed the laboratory analyses; R. E. and R. L.-R. supervised the dietary interventions and sampling; J. R., A. V.-Q. and R. L.-R. performed the statistical analyses. All authors were involved in data interpretation and manuscript preparation; R.L. had primary responsibility for the final content. All authors read and approved the final manuscript.

None of the authors has any conflict of interest.

\section{References}

1. Nielsen SE, Freese R, Kleemola P, et al. (2002) Flavonoids in human urine as biomarkers for intake of fruits and vegetables. Cancer Epidemiol Biomarkers Prev 11, 459-466.

2. Hu FB, Rimm E, Smith-Warner SA, et al. (1999) Reproducibility and validity of dietary patterns assessed with a food-frequency questionnaire. Am J Clin Nutr 69, 243-249.

3. Spencer JPE, Abd El Mohsen MM, Minihane A-M, et al. (2008) Biomarkers of the intake of dietary polyphenols: strengths, limitations and application in nutrition research. Br J Nutr 99, 12-22.

4. Ortuño J, Covas M-I, Farre M, et al. (2010) Matrix effects on the bioavailability of resveratrol in humans. Food Chem $\mathbf{1 2 0}$, 1123-1130.

5. Lehtonen H-M, Lindstedt A, Järvinen R, et al. (2013) $1 \mathrm{H}$ NMR-based metabolic fingerprinting of urine metabolites after consumption of lingonberries (Vaccinium vitis-idaea) with a high-fat meal. Food Chem 138, 982-990.

6. Hedrick V, Dietrich A, Estabrooks P, et al. (2012) Dietary biomarkers: advances, limitations and future directions. Nutr J 11, 109.

7. Zamora-Ros R, Urpí-Sardà M, Lamuela-Raventós RM, et al. (2009) Resveratrol metabolites in urine as a biomarker of wine intake in free-living subjects: the PREDIMED Study. Free Radical Biol Med 46, 1562-1566.

8. Zamora-Ros R, Urpí-Sardà M, Lamuela-Raventós RM, et al. (2006) Diagnostic performance of urinary resveratrol metabolites as a biomarker of moderate wine consumption. Clin Chem 52, 1373-1380. 
9. Romero-Pérez AI, Lamuela-Raventós RM, Waterhouse AL, et al. (1996) Levels of cis- and trans-resveratrol and their glucosides in white and rose Vitis vinifera wines from Spain. J Agric Food Chem 44, 2124-2128.

10. Romero-Pérez AI, Lamuela-Raventós RM, Buxaderas S, et al. (1996) Resveratrol and piceid as varietal markers of white wines. J Agric Food Chem 44, 1975-1978.

11. Bode LM, Bunzel $\mathrm{D}$, Huch $\mathrm{M}$, et al. (2013) In vivo and in vitro metabolism of trans-resveratrol by human gut microbiota. Am J Clin Nutr 97, 295-309.

12. Regmi U, Palma M \& Barroso CG (2012) Direct determination of organic acids in wine and wine-derived products by Fourier transform infrared (FT-IR) spectroscopy and chemometric techniques. Anal Chim Acta 732, $137-144$.

13. Ribéreau-Gayon P, Glories Y, Maujean A, et al. (2006) Organic acids in wine. In Handbook of Enology, pp. 1-49. New York: John Wiley \& Sons.

14. Velioglu Y (2009) Food acids: organic acids, volatile organic acids, and phenolic acids. In Advances in Food Biochemistry, pp. 313-339 [F Yildiz, editor]. Boca Raton, FL: CRC Press.

15. Spiller GA, Story JA, Furumoto EJ, et al. (2003) Effect of tartaric acid and dietary fibre from sun-dried raisins on colonic function and on bile acid and volatile fatty acid excretion in healthy adults. Br J Nutr 90, 803-807.

16. Lord RS, Burdette CK \& Bralley JA (2005) Significance of urinary tartaric acid. Clin Chem 51, 672-673.

17. Vázquez-Fresno R, Llorach R, Alcaro F, et al. (2012) 1H-NMRbased metabolomic analysis of the effect of moderate wine consumption on subjects with cardiovascular risk factors. Electrophoresis 33, 2345-2354.

18. Regueiro J, Vallverdu-Queralt A, Simal-Gandara J, et al. (2013) Development of a LC-ESI-MS/MS approach for the rapid quantification of main wine organic acids in human urine. J Agric Food Chem 61, 6763-6768.

19. Schulz KF, Altman DG \& Moher D (2010) CONSORT 2010 Statement: updated guidelines for reporting parallel group randomised trials. BMJ 340, c332.

20. Boeniger MF, Lowry LK \& Rosenberg J (1993) Interpretation of urine results used to assess chemical exposure with emphasis on creatinine adjustments: a review. Am Ind Hyg Assoc J 54, 615-627.

21. Medina-Remón A, Barrionuevo-González A, Zamora-Ros R, et al. (2009) Rapid Folin-Ciocalteu method using microtiter 96-well plate cartridges for solid phase extraction to assess urinary total phenolic compounds, as a biomarker of total polyphenols intake. Anal Chim Acta 634, 54-60.

22. Burns J, Yokota T, Ashihara H, et al. (2002) Plant foods and herbal sources of resveratrol. I Agric Food Chem 50, $3337-3340$.

23. Sobolev VS \& Cole RJ (1999) Trans-resveratrol content in commercial peanuts and peanut products. J Agric Food Chem 47, 1435-1439.

24. Flores P, Hellín P \& Fenoll J (2012) Determination of organic acids in fruits and vegetables by liquid chromatography with tandem-mass spectrometry. Food Chem 132, 1049-1054.

25. Blanck HM, Bowman BA, Cooper GR, et al. (2003) Laboratory issues: use of nutritional biomarkers. J Nutr 133, $888 \mathrm{~S}-894 \mathrm{~S}$.

26. Marshall JR (2003) Methodologic and statistical considerations regarding use of biomarkers of nutritional exposure in epidemiology. J Nutr 133, 881S-887S.

27. Lord RS, Burdette CK \& Bralley JA (2004) Urinary markers of yeast overgrowth. Integr Med 3, 24-29.

28. International Organisation of Vine and Wine (2012) Statistical Report on World Vitiviniculture 2012. Paris. http:// www.oiv.int/oiv/files/0\%20-\%20Actualites/EN/Report.pdf

29. Hunter D (1990) Biochemical indicators of dietary intake. In Nutritional Epidemiology, pp. 143-216 [W Willett, editor]. New York: Oxford University Press.

30. Roldán A, Palacios V, Caro I, et al. (2010) Evolution of resveratrol and piceid contents during the industrial winemaking process of sherry wine. J Agric Food Chem 58, 4268-4273.

31. Chadwick VS, Vince A, Killingley M, et al. (1978) The metabolism of tartrate in man and the rat. Clin Sci Mol Med $\mathbf{5 4}$ $273-281$.

32. Freedman LS, Tasevska N, Kipnis V, et al. (2010) Gains in statistical power from using a dietary biomarker in combination with self-reported intake to strengthen the analysis of a diet-disease association: an example from CAREDS. Am J Epidem 172, 836-842. 